\title{
FABP4 contributes to renal interstitial fibrosis via mediating inflammation and lipid metabolism
}

Yujie Qiao', Liping Liu², Lianhong Yin', Lina Xu', Zeyao Tang', Yan Qi', Zhang Mao', Yanyan Zhao' Xiau nng N a' and Jinyong Peng ${ }^{1,3,4}$

\section{Abstract}

Fatty acid binding protein 4 (FABP4), a subtype of fatty acid-binding protein family s s ws critical roles in metabolism and inflammation. However, its roles on regulating renal interstitial fibrosis (RIF) remair, nclear. In this work, LPSstimulated in vitro models on NRK-52E and NRK-49F cells, and in vivo UUO moa in rats and mice were established. The results showed that comparing with control groups or sham groups, $\mathrm{c}$ inels of a-SMA, COL1A, COL3A, IL-1ß, IL-6, and TNF-a in LPS-stimulated cells or UUO animals were sig fcantly increased. Meanwhile, the levels of TC, TG, and free fatty acid were also significantly increased as $V$ the covious lipid droplets, and the serum levels of BUN, Cr were significantly increased with large amounts of collazg, deposition in renal tissues. Further investigation showed that compared with control groups or sham grou os, the expression levels of FABP4 in LPSstimulated cells and UUO animals were significantly increasea, ulting in down- regulating the expression levels of PPARY, upregulating the levels of p65 and ICAM-1, and a reasin the expression levels of ACADM, ACADL, SCP-2, CPT1, EHHADH, and ACOX1. To deeply explore the prechan. of FABP4 in RIF, FABP4 siRNA and inhibitor interfered cell models, and UUO model on FABP4 knockout D) nicew whe used. The results showed that the expression levels of a-SMA, COL1A, and COL3A were significantry deo sed the deposition of lipid droplets decreased, and the contents of TC, TG, and free fatty acids wer, innifican decreased after gene silencing. Meanwhile, the expression levels of PPAR- $\gamma$, ACADM, ACADL, SCP-2, CRT1, $-\mathrm{HADH}$, and ACOX1 were upregulated, the levels of p65 and ICAM-1 were downregulated, and the mRNA evels of IL-1 $\mathrm{F}, \mathrm{IL}-6$, and TNF-a were decreased. Our results supported that FABP4 contributed to RIF via promoting in ammation and lipid metabolism, which should be considered as one new drug target to treat RIF.

\section{Introduction}

Renal fibrosis, a amn patnological process during the progression of onic kia ey disease (CKD) to the end stage renal disease FSRD), includes glomerular sclerosis (GS) and renal inter, atial fibrosis (RIF), in which renal

\section{Corres, idence. \\ Edited by R A Knight \\ (c) The Author(s) 2019}

1.

Depa Univers,y, 116011 Dalian, China

Full list of author information is available at the end of the article

These authors contributed equally: Yujie Qiao, Liping Liu interstitial lesions are more important than glomerular lesions to demonstrate the severity of renal function decline and prognostic prediction ${ }^{1}$. RIF with the accumulation of collagen components in renal interstitium can be caused by various pathogenic factors including glomerulonephritis, chronic pyelonephritis, obstructive nephropathy, diabetic nephropathy, hypertensive nephropathy, and kidney transplantation ${ }^{2}$. Thus, RIF, an important global public health issue, can seriously threaten human health and bring great economic burden to families and society.

At present, the detailed molecular mechanisms of RIF are not completely clarified. Multiple pathophysiological

(c) (i) Open Access This article is licensed under a Creative Commons Attribution 4.0 International License, which permits use, sharing, adaptation, distribution and reproduction cc) in any medium or format, as long as you give appropriate credit to the original author(s) and the source, provide a link to the Creative Commons license, and indicate if changes were made. The images or other third party material in this article are included in the article's Creative Commons license, unless indicated otherwise in a credit line to the material. If material is not included in the article's Creative Commons license and your intended use is not permitted by statutory regulation or exceeds the permitted use, you will need to obtain permission directly from the copyright holder. To view a copy of this license, visit http://creativecommons.org/licenses/by/4.0/. 
changes including inflammation, apoptosis and oxidative stress can cause fibrosis. Recent studies have found that massive proteinuria can cause the overload of free fatty acids (FFAs) in renal interstitial cells and lipid hydroperoxides after oxidization in mitochondria and lysosomes ${ }^{3}$. The molecules associated with reactive oxygen species (ROS) can destroy cell membrane, and cause severe renal interstitial damage and fibrosis. It has also been found that increased absorption of FFAs can result in apoptosis and damage of renal interstitial cells ${ }^{4}$. Under normal conditions, fatty acid oxidation can produce energy for renal tubular epithelial cells. However, reduced fatty acid metabolism can cause ATP depletion, cell death, lipid accumulation, and ultimately lead to RIF. On the other hand, transforming growth factor- $\beta$ (TGF- $\beta$ ) can reduce fatty acid oxidation in renal tubular epithelial cells to promote renal fibrosis ${ }^{5}$. In addition, macrophages, the predominant infiltrating immune cells, can produce various proinflammatory cytokines, which are closely associated with renal fibrosis ${ }^{6}$. Monocyte chemoattractant protein-1 (MCP-1), an important proinflammatory cytokine, has important role in the progression of tubulointerstitial fibrosis ${ }^{7}$. Thus, regulating lipid metabolism and inflammation should be one effective method to control RIF.

Fatty acid-binding protein 4 (FABP4), a subtype of fatty acid-binding protein family, is a key transmitter lip $\mathrm{d}$ metabolism and inflammatory reaction ${ }^{8}$. FABP $\mathrm{r}$ is $\mathrm{P}$ tively correlated with FFAs, and high level o. 4BP4 ca. directly damage endothelial cells, while tho njured endothelial cells can promote FABPA level, folloved by deposition of triglyceride and chole erol, together with lipid metabolism disorders ${ }^{9}$. It has on reported that peroxisome proliferator activat 'receptor (PPAR $\gamma$ ), one target gene of FABP4, can be negatr edback controlled by FABP $4^{10}$. However, $R \gamma$ is able to decrease NF- $k B$ activity, which can at eft atively inhibit the expression levels of intercelly.ar co dhesion molecule-1 (ICAM-1) and vascular adhes 1 molecule 1 (VCAM- 1$)^{11}$. Activated $\mathrm{PP} \wedge \mathrm{R} \gamma$ suppress the production of tumor necrosis factor- $\alpha$ (TN - $\alpha$ ), Interleukin-1 (IL-1), IL-4, and IL-6 wh ar inflammatory effect ${ }^{12}$. Meanwhile, PPAR $\gamma$ can mula the processes of fatty acid transport, oxidaon hd de,omposition by regulating the expression lc 1 or _...ty acid transporter, fatty acid binding protein, and nitine palmitoyltransferase- $1(\mathrm{CPT} 1)^{13}$. In addition, FABP4 can adjust the eicosanoid balance by regulating the activities of cyclooxygenase 2 (COX2) and leukotriene A4 (LTA4), and ultimately affect the functions of macrophages and inflammation ${ }^{14}$. Moreover, FABP4 can regulate obesity-induced neuroinflammation through FABP4-uncoupling protein 2 (UCP2) axis ${ }^{15}$. In diabetic nephropathy, FABP4 can regulate apoptosis of renal interstitial cells via adjusting endoplasmic reticulum stress, which may also serve as a marker of renal injury ${ }^{16}$. In addition, inhibition of FABP4 can reduce hepatic liver ischemia- reperfusion injury ${ }^{17}$. Hence, FABP4 plays critical roles in regulating inflammation and lipid metabolism. However, there have no studies concerning the roles of FABP4 in RIF. Therefore, the aim of the presen work was to investigate the function of $F A B P A$ in regulating RIF.

\section{Materials and methods \\ Chemicals and materials}

Dulbecco's Modified Eagle's I edium (D, /EM) was purchased from KeyGEN (Jiangsu Thina). The assay kits of blood urea nitrogen (BUK, reat. $(\mathrm{Cr})$, total cholesterol (TC), triglyceridf (TG), a 'free fatty acids (FFAs) were obtained from va. ng Jiancheng Institute of Biotechnology (Nanjing, Chin Tissue Protein Extraction Kit and the Bicincr hinic acid (BCA) Protein Assay Kit were purchasea time Institute of Biotechnology (Jiangsu, China). sodium dodecyl sulfate (SDS) and 4,6-diami 2-phenylindole (DAPI) were purchased from Sigma (So, Louis, MO, USA). Lipopolysaccharide (LPS) and $\mathrm{bil}_{\mathrm{i}}$ red $\mathrm{O}$ staining solution were purchased tro Solarbio Technology Co., Ltd (Beijing, China) and Keyc N (Jiangsu, China). CCK-8 was purchased from 'er. $\mathrm{k}$ Chemicals (Houston, USA). Lipofectamine 2000 wàs.s obtained from GenePharma (Shanghai, China). RNAisoPlus, PrimeScript ${ }^{\oplus} \mathrm{RT}$ reagent Kit with DNA Eraser (Perfect Real Time) and SYBR ${ }^{\oplus}$ Premix Ex TaqTMII (Tli RNaseH Plus) were purchased from TaKaRa Biotechnology Co., Ltd. (Dalian, China). FABP4 siRNA was purchased from RIBOBIO Co., Ltd. (Guangzhou, China). Mouse and Rat FABP4 ELISA Kits were purchased from Boster Biological Technology Co., Ltd. (California, USA). FABP4 inhibitor BMS309403 was purchased from MedChemExpress (Shanghai, China).

\section{Cell culture}

NRK-52E cell line (a rat proximal tubular epithelial cell) and NRK-49F cell line (a kind of rat fibroblast) were purchased from the Institute of Biochemistry Cell Biology (Shanghai, China) and BeNa Culture Collection (Beijing, China), which were maintained in DMEM or MEM NEAA supplemented with $10 \%$ FBS and antibiotics (100 IU/mL penicillin and $100 \mathrm{mg} / \mathrm{mL}$ streptomycin) in a humidified atmosphere of $5 \% \mathrm{CO}_{2}$ and $95 \% \mathrm{O}_{2}$ at $37^{\circ} \mathrm{C}$.

\section{LPS-induced cell proliferation}

NRK-52E and NRK-49F cells were plated in 96-well plates at a density of $1 \times 10^{5}$ cells $/ \mathrm{mL}$ for $24 \mathrm{~h}$ before challenge with various concentrations of LPS $(0,25,50$, $100,200$, and $400 \mathrm{ng} / \mathrm{mL})$ for different times $(3,6,12$, and $24 \mathrm{~h})$. Then, CCK-8 solution was added to the plates for $4 \mathrm{~h}$ incubation at $37^{\circ} \mathrm{C}$, and the absorbance was measured 
at $450 \mathrm{~nm}$ with a microplate reader (Thermo, Waltham, MA, USA).

\section{Unilateral ureteral obstruction (UUO) model on rats and mice}

Male Sprague Dawley rats (180-220 g) and Male C57BL/6J mice (18-22 g) were purchased from the Experimental Animal Center of Dalian Medical University (Dalian, China) (SCXK (Liao): 2013-0003). FABP4 knockout (KO) and WT mice (20-22 g) were purchased from Nanjing Biomedical Research Institute of Nanjing University (Nanjing, China) (SCXK (Su): 2015-0001). The animals were maintained in a controlled environment under standard conditions with temperature at $21-25^{\circ} \mathrm{C}$ and relative humidity at $55-70 \%$. The animals were provided with ample food and water and maintained on a $12 \mathrm{~h} / 12 \mathrm{~h}$ light-dark cycle. The rats and mice were randomly divided into sham group and UUO model group $(n=7)$. FABP4 KO and WT mice were randomly divided into sham group of WT mice, UUO model group of WT mice, sham group of FABP4-KO mice, and UUO model group of FABP4-KO mice $(n=5)$. The animals in UUO groups were achieved by ligating the left ureter with 3-0 silk through a left lateral incision. The animals in sham-operated groups were used as the control. After 4 weeks of rats and 7 days of mice ${ }^{18,19}$, the animals vere sacrificed. The blood samples were obtained to $p$ du serum after centrifugation $\left(1200 \times g, 4{ }^{\circ} \mathrm{C}\right)$, w'ich stored at $-20^{\circ} \mathrm{C}$. The kidney samples we harveste, and stored at $-80^{\circ} \mathrm{C}$.

\section{Assessment of biochemical paramete}

The protein samples from NRK-5 cells and NRK49F cells were extracted using ald lysis ouffer containing $1 \mathrm{mM}$ phenylmethyl sulforyl ide according to the protocol, and the ents were determined using BCA Protein Assay $\mathrm{TH}$ level, of TC, TG, and FFAs in cell lysates wae is sured using the commercial kits $^{20}$. The ser a levels o, $₫$ UN, Cr, TC, TG, and FFAs of rats and mice $m$ also detected using the commercial kits acco-ding to the nanufacturer's instructions.

ELIcn assa

The serum protein levels of FABP4 in rats and mice $n$ musured using the assay kits according to the man ycturer's instructions ${ }^{21}$.

\section{Histological assay}

Formalin-fixed renal tissue was embedded in paraffin. The portion of renal cortex was separated and fixed in formaldehyde (10\%), and then the section with $5 \mu \mathrm{m}$ thick was stained with H\&E, Masson, Sirius red staining assays. Images were acquired and the histological evaluations were performed using a light microscope (Nikon Eclipse
TE2000-U, NIKON, Japan) with 400× magnification. In addition, the images of Sirius red polarization assay were captured using a polarized light microscope (NIKON Eclipse Ci, NIKON, Japan) with 400× magnification.

\section{Oil Red $\mathbf{O}$ staining of cells and renal tissues}

The frozen tissue slices or formal in-fixed $c$ lls were washing with PBS, and then Oil red O solution $\mathrm{m}$ aded and incubated at room temperature in the dat for $30 \mathrm{~min}$, which were finally washed with \% isopi opanol and PBS. Then, the images wer capt $r$ asing a microscope (Olympus, Tokyo, Japan), y/th 400x magnification.

\section{Immunofluorescence ass $y$}

For immunofluores ce stainiyg of FABP4, $\alpha$-SMA, and COL1A, the tissue slice $r$ formal in-fixed cells were incubated with a i-FABPs, anti- $\alpha$-SMA, and antiCOL1A antib ectively, in one humidified chamber at $4{ }^{\circ} \mathrm{C}$ on vight, followed by incubation with an alexa fluo in-labeled secondary antibody at $37^{\circ} \mathrm{C}$ for $1 \mathrm{~h}$. Eventual $/ y$, il nuclei were stained with DAPI (5 mg/ $\mathrm{mL}$ ) for $15 \mathrm{nin}$. Then, the immune stained images were $\mathrm{Ca}_{\mathrm{F}}$ red using a fluorescence microscope (Olympus, Toky Japan) with $400 \times$ magnification.

\section{W'stern blot assay}

Total protein samples from kidney tissues of rats and mice, NRK-52E and NRK-49F cells were extracted using cold lysis buffer containing $1 \mathrm{mM}$ phenylmethyl sulfonyl fluoride according to the manufacturer's protocol, and the protein content was determined using the BCA protein assay kit. The protein samples were loaded onto SDSPAGE gel (8-12\%), electrophoretically separated and transferred onto a PVDF membrane (Millipore, USA). After blocking nonspecific binding sites for $1 \mathrm{~h}$ with $5 \%$ dried skim milk in TTBS at $37^{\circ} \mathrm{C}$, the membrane was individually incubated for overnight at $4{ }^{\circ} \mathrm{C}$ with the primary antibodies listed in Supplemental Table S1. Then, the membrane was incubated at room temperature for $2 \mathrm{~h}$ with horseradish peroxidase-conjugated antibodies at a 1:2000 dilution. Protein expression was detected by the enhanced chemiluminescence (ECL) method. Protein bands were imaged using a Bio-Spectrum Gel Imaging System (UVP, Upland, CA, USA) and normalized with GAPDH as an internal control (IOD of objective protein versus IOD of GAPDH protein).

\section{Quantitative real-time PCR assay}

Total RNA samples were obtained from kidney tissues, NRK-52E, and NRK-49F cells using RNAiso Plus reagent following the manufacturer's protocol. Each RNA sample was reverse transcribed into cDNA using the PrimeScript1 RT reagent Kit. The forward (F) and 
reverse (R) primers used in the present study are given in Supplemental Table S2. Among the data from each sample, the $\mathrm{Ct}$ value of the target genes was normalized to that of GAPDH. The unknown template in our study was calculated using the standard curve for quantitative analysis.

\section{FABP4 siRNA treatment}

FABP4-targeted siRNA and control siRNA were dissolved in DMEM or MEM NEAA and then equilibrated for $5 \mathrm{~min}$ at room temperature. NRK-52E and NRK-49F cells were transfected with FABP4 siRNA or non-binding control siRNA using Lipofectamine 2000 reagent according to the protocol. Then, the levels of TC, TG, and FFAs were detected. Oil Red $\mathrm{O}$ staining and immunofluorescence assay were carried out for detecting lipid droplets and the expression levels of $\alpha$-SMA, COL1A, and FABP4 after transfection. In addition, the protein levels of FABP4, PPAR $\gamma$ SCP2, ACADM, ACADL, EHHADH, CPT1, ACOX1, p65, ICAM-1, and the mRNA levels of $\alpha-$ SMA, COL1A, COL3A, IL-1 $\beta$, IL- 6 , and TNF- $\alpha$ were measured after transfection.

\section{Statistical analysis}

The data and statistical analysis in this study comply with the recommendations on experimental design and analysis in pharmacology. The data are expressed as the mean \pm SD. GraphPad Prism 6.01 software (Paragraph Software, Inc, La Jolla, CA, USA) was used to handl these data and only when a minimum of $n=5$ ind pendent samples was acquired. Statistical significance wc etermined by one-way ANOVA. Analysis between two $1-$ vidual groups was determined by Stu t $t$-te the The results were considered to be statistifanry sig. cart at $p<$ 0.05 .

\section{Results}

Fibrosis and lipid metabr,ilsm dis. Jers in cells caused by LPS

As shown in Fig. 1a, the vpression levels of $\alpha$-SMA, COL1A1, and COL 11 were significantly increased $(p<$ 0.01 ) in NRK -5 a $\quad$ K-49F cells after LPS treatment compared with a trol groups. (The survival rates of NRK-52E NRK-49F treated by LPS are shown in Supplemer ta $/ \mathrm{F}_{1}, \mathrm{~S}$ ). Based on these results, LPS at the




concentration of $200 \mathrm{ng} / \mathrm{mL}$ for NRK52E cells, and $25 \mathrm{ng} /$ $\mathrm{mL}$ for NRK49F cells under $3 \mathrm{~h}$ treatment were selected in the rest of the experiments. The results of immunofluorescence assay also showed the high expression levels of $\alpha$-SMA (green light) and COL1A (red light) in LPSstimulated cells. In addition, as shown in Fig. 1b, Oil red $\mathrm{O}$ staining revealed that lipid droplets were accumulated in NRK-52E and NRK-49F cells after LPS treatment compared with control groups. Furthermore, the levels of TC, TG and FFAs were significantly increased by 38.76 , 79.57, and $85.73 \%$ in LPS-induced NRK-52E cells compared with un-treated cells, and increased by $30.97,70.07$, and $85.16 \%$ in LPS-induced NRK-49F cells compared with control group.

\section{Renal injury and histopathological changes in UUO rats and mice}

As shown in Fig. 1c, compared with sham groups, the levels of serum $\mathrm{Cr}$ (mice $25.506 \pm 4.596 \mu \mathrm{mol} / \mathrm{L}$; rats $53.344 \pm 16.458 \mu \mathrm{mol} / \mathrm{L}$ ) and BUN (mice $10.833 \pm$ $1.803 \mathrm{mmol} / \mathrm{L}$; rats $8.333 \pm 1.883 \mathrm{mmol} / \mathrm{L}$ ) in UUO animals were significantly increased. The results of $H \& E$ staining showed that the kidneys of the animals in sham groups exhibited integral tubular cell structure. However, the histopathological changes including swelling in renal tubular epithelial cells, vacuoles degeneration, disapreating of brush border, coagulation necrosis, and si ? inflammatory cells infiltration in UUO anir $1 \mathrm{l} s$, e obviously found compared with sham grour.

\section{UUO induces RIF and lipid metabolism disorders}

As shown in Fig. 1d, compared wi sham groups, the interstital and perivascular collager lepositions were obviously found in UUO ani ${ }^{1}$, , ana the expression levels of COL1A (green) and CPL, orange red) were heavily increased comp with sham groups, as well as the results of polarize ligh nher ation. As shown in Fig. 1e, the mRNA leveru of "MA, COL1A1, and COL3A1 in UUO animals re mark ily increased compared with sham group. In dition, immunofluorescence assay showed that the exps ssion levels of $\alpha$-SMA (green) and COL1h in UUO animals were markedly increased commared th sham groups. As shown in Fig. 1f, Oil Red sta ing in alcated that the lipid droplets in renal tissue h sig....cantly increased in UUO animals. At the same time ompared with sham groups, the levels of TC, TG, and PFAs were significantly increased by $21.19,29.43$, and $40.38 \%$ in UUO mice, and increased by $44.74,61.81$, and $34.38 \%$ in UUO rats.

\section{Expression levels of FABP4 in RIF}

As shown in Fig. 2a, b, in LPS-induced cells, the expression levels of FABP4 were significantly increased compared with control groups. Similarly, in UUO rats and mice, the expression levels of FABP4 were significantly increased compared with sham groups (Fig. 2c, d). In addition, the serum protein levels of FABP4 in UUO rats and mice were significantly increased with $p<0.01 \mathrm{com}$ pared with sham groups (Fig. 2e).

\section{FABP4 adjusts PPARy to regulate inflammation > d fatty acid oxidation}

As shown in Fig. 3a, the protein levels of PPARY in ofstimulated cells or UUO animals signi scantly reduced compared with control grsups or $\mathrm{nm}$ /groups in vitro and in vivo. As shown in $\mathrm{F}$. $3 \mathrm{~b}$, the $\mathrm{m}, \mathrm{NA}$ levels of IL-1 $\beta$, IL-6, and TNF- $\alpha$ ir L -stimy lated cells or UUO animals were significan incru and the protein levels of p65 and ICAM-1 were a significantly increased compared with contre $\mathrm{s}$ ups or siam groups. As shown in Fig. 3c, the expression els of some proteins associated with facty cid oxidation including ACADL, ACADM, CP 1 , SCP-2, and EHHADH were significantly decre d, in LPS-treated cells or UUO animals con wi control groups or sham groups (Details of fola y ranges and significances of the proteins in western blot assay are shown in Supplemental Fig. S2).

FABP siRNA inhibits inflammation and reinforces PPARY - na/in vitro

As shown in Fig. 4a, the expression levels of FABP4 were massively reduced in FABP4 siRNA groups compared with LPS-stimulated cells. Besides, western blotting assay illustrated that the expression levels of FABP4 were markedly decreased in FABP4 siRNA groups compared with LPS-stimulated cells. In contrast, the expression levels of PPAR $\gamma$ in FABP4 siRNA-treated NRK-52E and NRK-49F cells were significantly increased compared with LPS-stimulated cells. In addition, compared with LPSstimulated cells, FABP4 siRNA suppressed inflammation by decreasing the mRNA levels of IL- $1 \beta$, IL- 6 , and TNF- $\alpha$ in NRK-52E and NRK-49F cells, and the protein levels p65 and ICAM-1 were also obviously reduced (Fig. 4b). Besides, the data in Fig. 4c showed that, compared with LPS-stimulated cells, FABP4 inhibition improved fatty acid oxidation via reinforcing PPAR $\gamma$ signal by affecting the protein levels ACADL, ACADM, CPT1, ACOX1, SCP-2, and EHHADH (Details of fold changes and significances of these proteins in western blot assay are shown in Supplemental Fig. S3).

\section{FABP4 siRNA attenuates fibrosis and lipid metabolism disorders in cells}

As shown in Fig. 4d, compared with LPS-stimulated cells, the mRNA levels of $\alpha$-SMA, COL1A1, and COL3A1 were significantly decreased in FABP4 siRNA groups. Meanwhile, FABP4 siRNA attenuated LPS-induced fibrosis in vitro by reducing the expression levels of $\alpha$-SMA (green) 

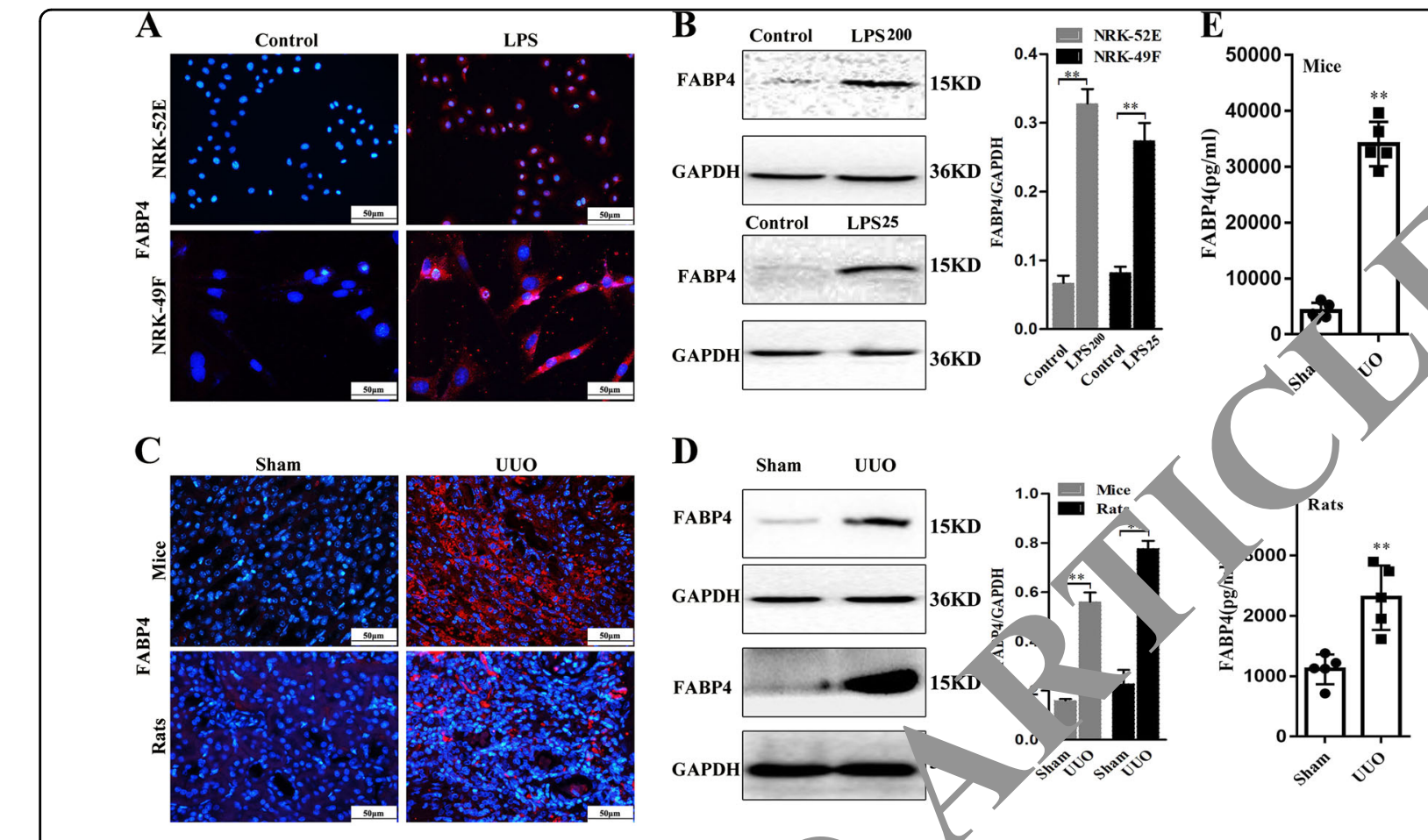

Fig. 2 Increased protein levels of FABP4 in RIF models. a, b The proteIn m of FABP4 in NRK-52E and NRK-49F cells based on immunofluorescence staining and western blotting assay $(n=3)$. $\mathbf{c}, \mathbf{d} /$ protein vels of FABP4 in mice and rats based on immunofluorescence staining and western blotting assay $(n=3)$. e Serum protein levels $\quad F A B$ mic and rats based on Elisa assay $(n=7)$. Data are presented as the mean \pm SD. ${ }^{* *} p<0.01$ compared with sham groups or control oups

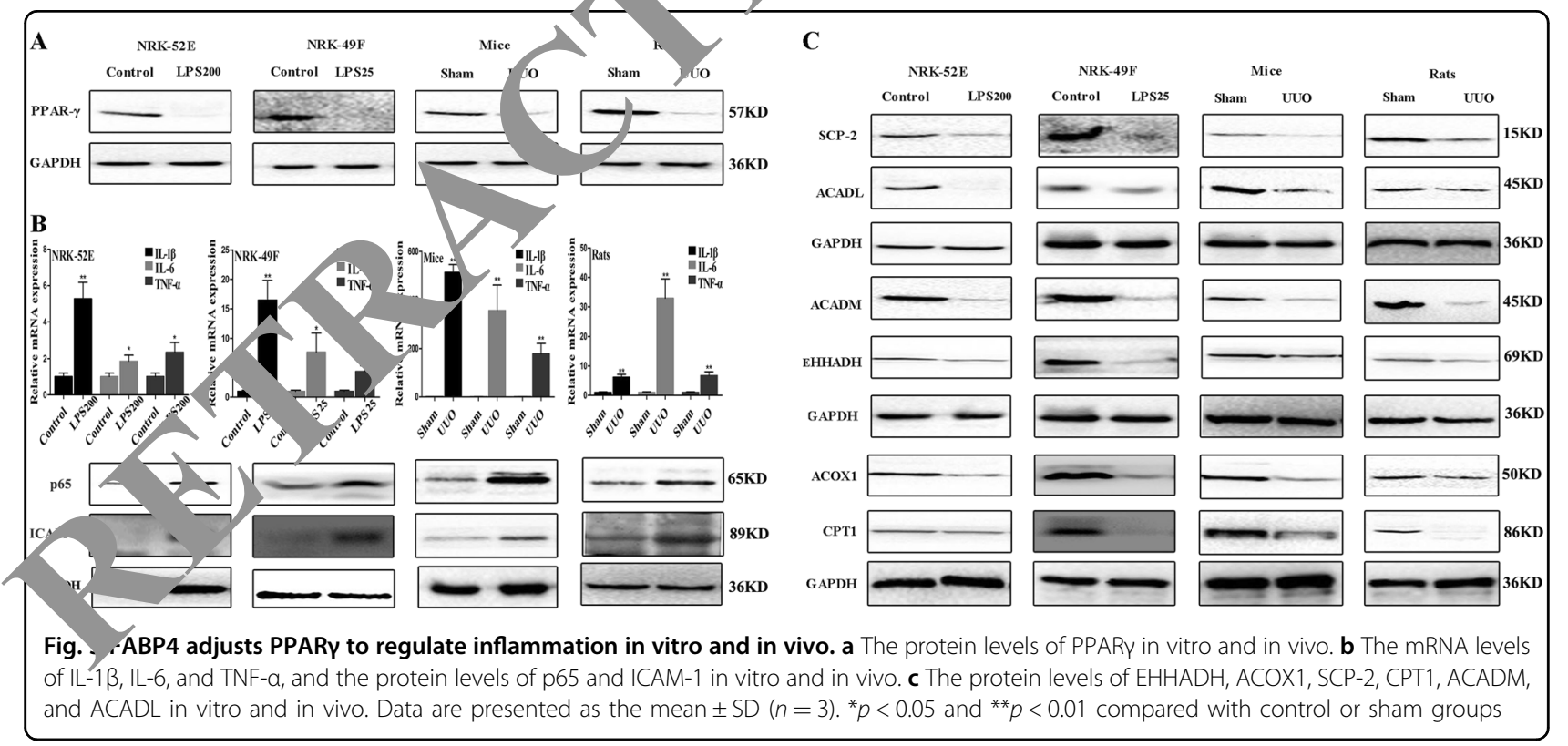

and COL1A (red). As shown in Fig. 4e, the levels of TC, TG, and FFAs in FABP4 siRNA-treated NRK-52E cells were reduced by $55.27,33.33$, and $42.13 \%$, and decreased by 51.01, 33.41, and 53.65\% in NRK-49F cells compared with
LPS-stimulated cells. Furthermore, the results of Oil Red O staining showed that the depositions of lipid droplets in FABP4 siRNA-treated cells were obviously reduced compared with LPS-stimulated cells. 


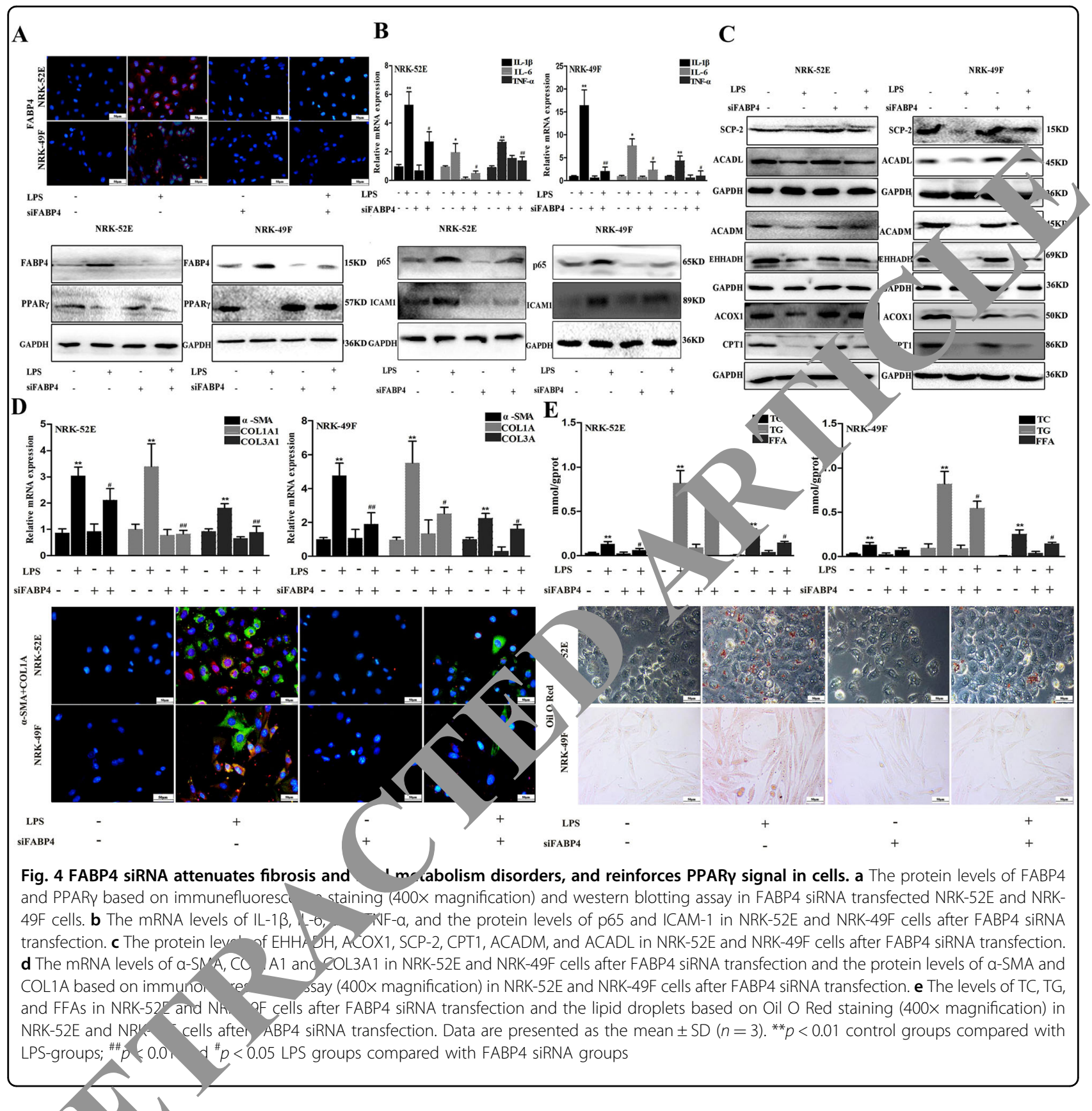

ABP. inhibi or decreases FABP4 expression, reinforces h. $r$ i $\ldots$. al, and reverses fibrosis and lipid metabolism diso. $r s$ in cells

As shown in Fig. 5a, the expression levels of FABP4, p65, and ICAM were decreased in FABP4 inhibitor groups, while the expression levels of PPAR $\gamma$ in FABP4 inhibitor-treated NRK-52E and NRK-49F cells were increased compared with LPS stimulated cells. The data in Fig. 5b showed that the expression levels of ACADL, ACADM, CPT1, ACOX1, SCP-2, and EHHADH in NRK-52E and NRK-49F cells were increased by FABP4 inhibitor compared with LPS-stimulated cells. In addition, as shown in Fig. 5c, the mRNA levels of $\alpha$-SMA, COL1A1 and COL3A1 were significantly decreased in FABP4 inhibitor groups and the expression levels of $\alpha$-SMA (green) and COL1A (red) were reduced compared with LPS groups $(p<0.05)$. Moreover, the results of Oil Red $\mathrm{O}$ staining in Fig. $5 \mathrm{~d}$ showed that the depositions of lipid droplets, and the levels of TC, TG, and FFAs in FABP4 inhibitor-treated cells were significantly decreased compared with LPS-stimulated cells $(p<0.05)$. 


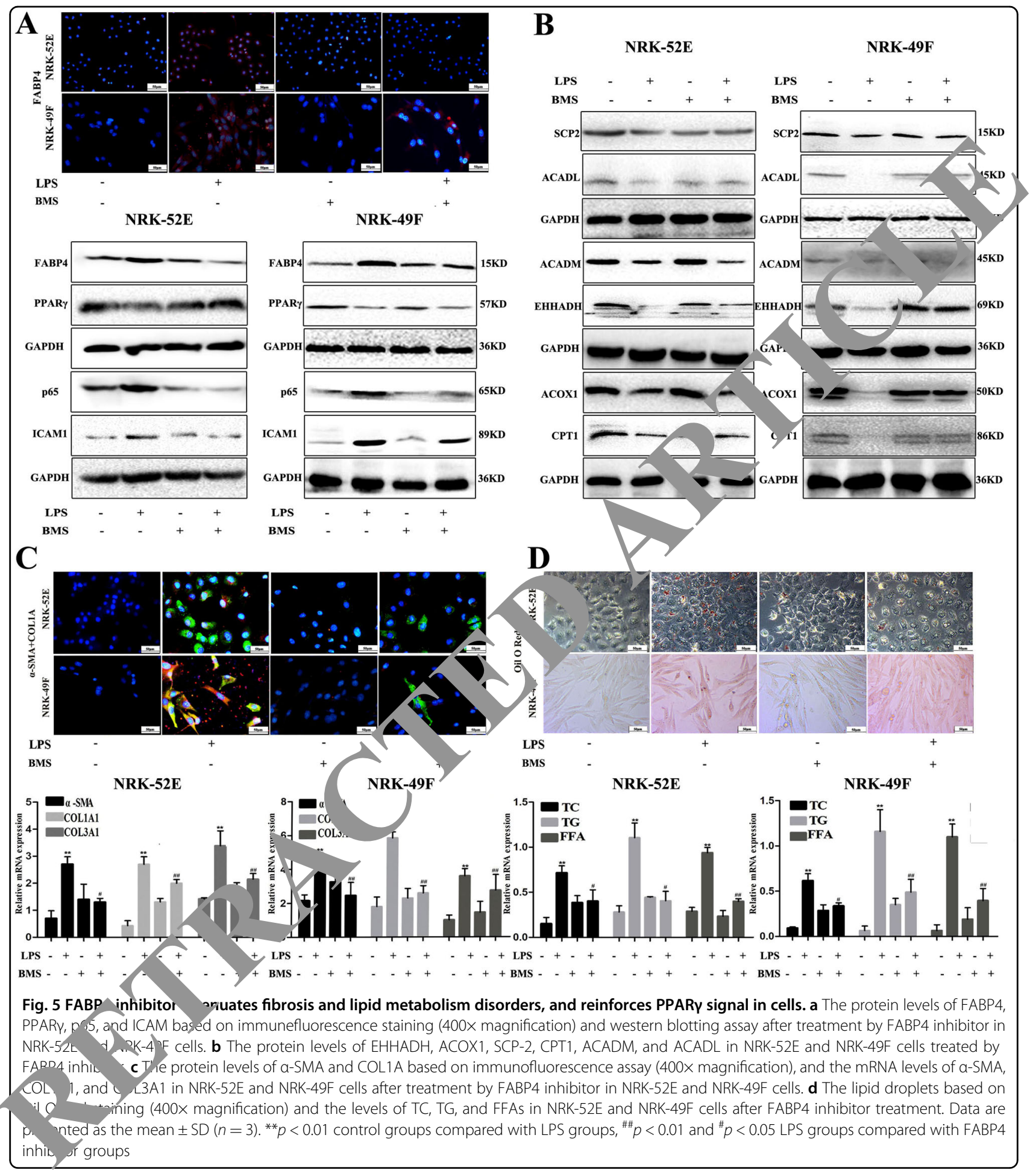

FABP4 KO downregulates FABP4 expression and reinforces PPARy signal in mice

As expected, the expression level of FABP4 in FABP4 $\mathrm{KO}$ mice was not detected (Fig. 6a). As shown in Fig. 6b, the expression level of PPAR $\gamma$ was significantly increased in UUO FABP4 $\mathrm{KO}$ mice compared with WT mice. In addition, compared with UUO WT animal, the mRNA levels of IL-1 $\beta$, IL- 6 , and TNF- $\alpha$ in UUO FABP4 KO mice were reduced, and the protein levels of p65 and ICAM-1 were also decreased (Fig. 6c, d). In addition, the data in Fig. 6d showed that, compared with UUO WT mice, FABP4 knockdown attenuated lipid metabolism disorders 


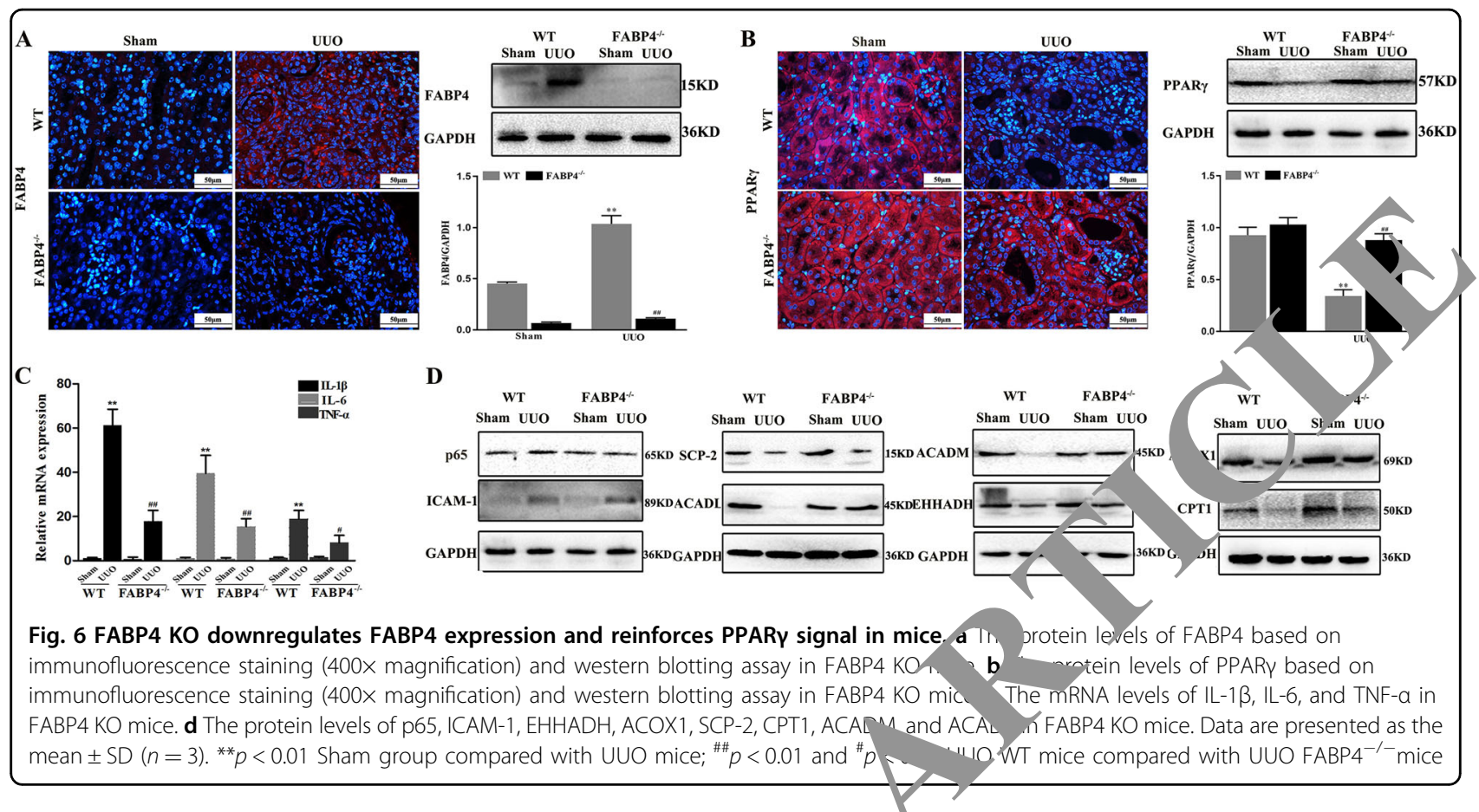

via inhibiting PPAR $\gamma$ signal by affecting the protein levels of ACADL, ACADM, CPT1, ACOX1, SCP-2, ana EHHADH in UUO FABP4 KO mice (Details of $10 \mathrm{dd}$ changes and significances of these proteins in $\mathrm{y}$ te blot assay are shown in Supplemental Fig. S4)

\section{FABP4 KO protects renal function in mice}

As shown in Fig. 7a, the levels of $\mathrm{Cr}$ (59,12 \pm $8.991 \mu \mathrm{mol} / \mathrm{L})$ and BUN $(13.720 \pm 297 \mathrm{mmol} / \mathrm{L})$ were significantly increased in UUO WT ice. However, in FABP4 KO mice after UUO o ration, the levels of $\mathrm{Cr}$ $(18.975 \pm 4.959 \mu \mathrm{mol} / \mathrm{L})$ and BUN $.7 \pm 1.316 \mathrm{mmol} / \mathrm{L})$ were significantly reduc The esults of $\mathrm{H} \& \mathrm{E}$ staining showed that in UU FA D4. KS mice, the extent of tubular dilation or a phy and the infiltration of inflammatory were arkedly improved compared with UUO W $\Gamma$ an

FABP4. רt nu tes RIF and lipid metabolism disorders in micn

Cor pared with UUO WT mice, collagen deposition h runced and fibrosis degree was significantly imp ad in UUO FABP4 KO mice (Fig. 7b). Under polarized light observation, the expression levels of COL1A (green) and COL3A (orange red) were heavily decreased in UUO FABP4 KO mice compared with UUO WT mice. At the same time, as shown in Fig. 7c, the mRNA levels of $\alpha$-SMA, COL1A1, and COL3A1 were significantly decreased in UUO FABP4 KO mice compared with UUO WT mice. In addition, Oil Red O staining observed that the deposition of lipid droplets was
Sly cantly reduced in FABP4 KO UUO mice compared with UO WT mice (Fig. 7d). Furthermore, the levels of $\mathrm{G}$, and FFAs were also significantly reduced by $18.14,48.28$, and $44.30 \%$.

\section{Discussion}

RIF is the pathological basis or pathological feature that leads to chronic renal failure, which is also the best histologic predictor of renal functional decline in $C K \mathrm{D}^{22}$. Therefore, it is necessary to explore effective drug targets for research and development of innovative drugs to treat RIF.

Recently, some works have reported that dyslipidemia associated with lipid metabolism disorder plays an important role in the pathogenesis of $\mathrm{RIF}^{23}$. Lemos et al. have observed that inhibiting Interleukin-1R (IL-1R) signal transducer kinase IRAK4 (Interleukin-1 receptorassociated kinase 4) can abrogate fibrosis and reduce tubular injury ${ }^{24}$. In our study, the survival rates of NRK$52 \mathrm{E}$ and NRK-49F after challenge with LPS were detected and UUO models in mice for 7 days and rats for 4 weeks were established ${ }^{25,26}$. The results showed that in vivo experiments, compared with sham groups, the levels of serum $\mathrm{Cr}$ and BUN in UUO animals were significantly increased $(p<0.05)$, as well as the histopathological changes. At the same time, the levels of inflammationrelated factors including IL-1 $\beta$, IL- 6 , and TNF- $\alpha$ were increased in UUO mice and rats. In addition, we also found that the levels of TC, TG, and FFAs were increased, and lipid depositions were obvious in UUO animals. The same results were verified in vitro experiments. Therefore, 


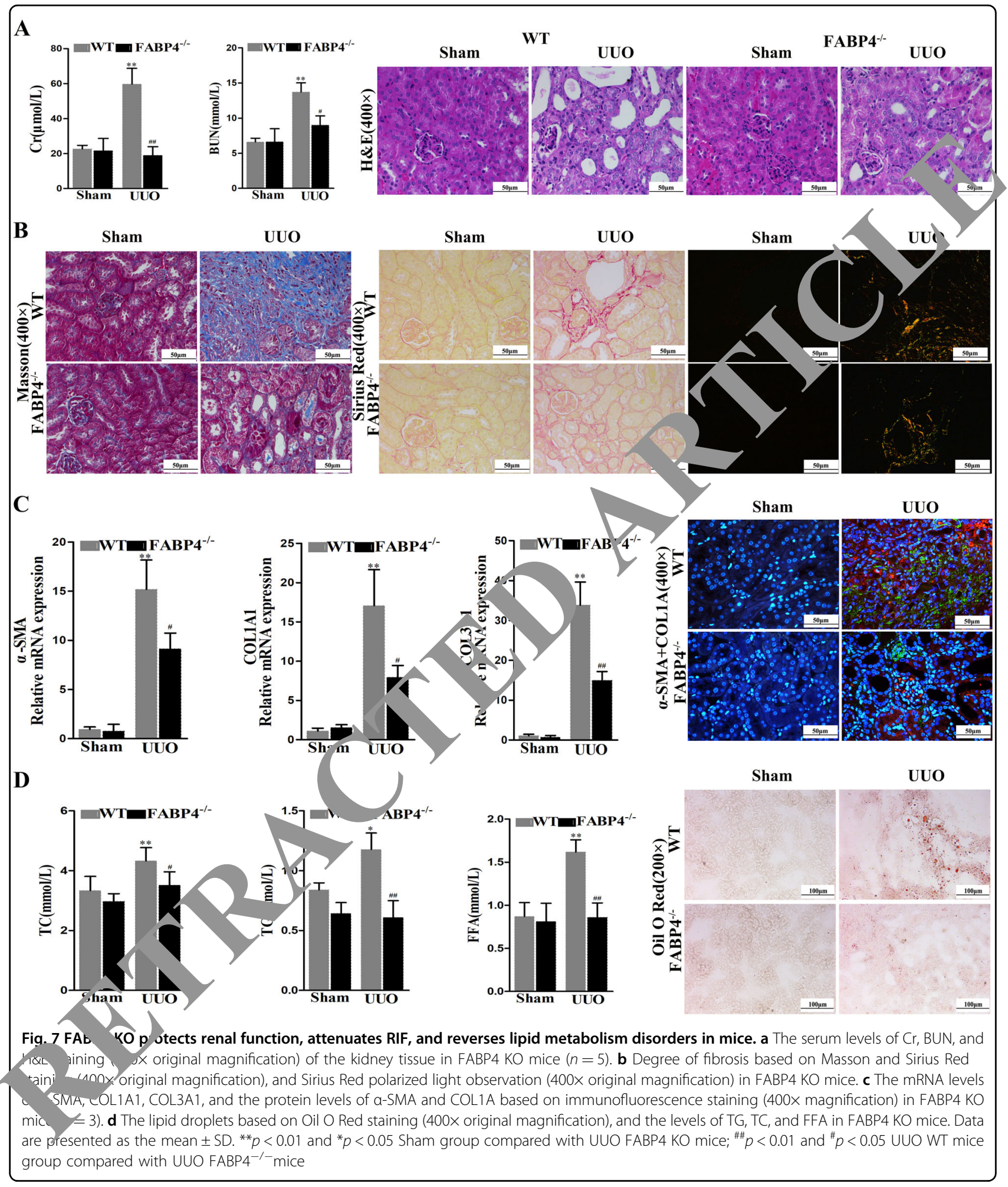

inflammation and lipid metabolism disorders played important roles in the process of RIF.

Therefore, it is important to find drug targets that can regulate inflammation and lipid metabolism to treat RIF.
FABP4, a member of the fatty acid-binding protein family, is involved in lipid metabolism and inflammation ${ }^{27,28}$. Recent studies have shown that FABP4 inhibitor can reduce lipid-induced ER stress-associated inflammation, 
ameliorate lipid deposits and suppress ROS and nuclear factor-kappaB (NF-kB) nuclear translocation ${ }^{29}$. In other hand, FABP4 deficiency can alter adipocyte biology and fatty acid metabolism to regulate systemic insulin resistance, dyslipidemia, and lipotoxicity ${ }^{30}$. However, the mechanisms of FABP4 in RIF via regulating inflammation and dyslipidemia remain poorly defined.

Some reports have shown that FABPs can correlate with PPAR $\gamma$, in which FABP4 can specifically connect with PPAR $\gamma^{31}$. Besides, in FABP4-deficient macrophages, the cholesterol accumulation and alterations in proinflammatory responsiveness can be suppressed ${ }^{32}$. At the same time, the shortage of FABP4 alters lipid composition in macrophages and enhances PPARY activity, leading to the elevated expression of CD36 and enhanced uptake of modified low density lipoprotein ${ }^{33}$. In our present study, the results showed that the occurrences of RIF were accompanied with the increased expression of FABP4 and the decreased expression of PPARy in vivo and in vitro experiments. Then, FABP4 siRNA and inhibitor interfered NRK-52E and NRK-49F cells, and FABP4 KO mice were used. We found that knocking down and inhibition of FABP4 promoted PPAR $\gamma$ expression. These data proved that downregulation of FABP4 increased PPAR $\gamma$ expression in feedback, as a result of reversing RIF.

Inflammation is a driver of RIF. PPAR $\gamma$ can regulate $\mathrm{NT}$ $\kappa \mathrm{B}$ activation, and activated PPAR $\gamma$ can be indy. IV FABP4, and then PPAR $\gamma$ binds to p65 in the cusleu ? prevent the activation of NF-кB-RE (Nuclear, tor kapp beta response element) during inflammation ${ }^{34}$, In the present study, FABP4 inhibition or kn ockdown inc, eased PPAR- $\gamma$ expression, and decreased 1 e protein levels of p65 and ICAM-1 to suppress inflam tion. These data indicated that suppression of $\mathrm{ABP} 4$ Innibited NF- $\mathrm{KB}$ activation to improve inflammatior inst RIF.

In recent years, a nu $r$ of nimal experiments and clinical studies have hov that lipid metabolism disorders can also plomerulosclerosis and tubulointerstitial in $\quad 36$. Somt esearchers have reported that FABP4 KO can pro nt obesity-induced insulin resistance and reduce the rate $o_{1}, 1$ polysis with normal diet of mice ${ }^{37}$. In the $\mathrm{P}$ sti dy, we found that FABP4 KO in mice or $\mathrm{FAPO}_{4} \mathrm{Sh}_{\mathrm{L}} \mathrm{A}$ and inhibitor in cells decreased lipid epos ion, a d reversed the levels of TC, TG, FFAs. By c. as, he expression levels of the proteins associated with "atty acid oxidation including SCP2, ACADL, ACADM, CPT1, ACOX1, and EHHADH were increased, suggesting that the mechanism of FABP4 in regulating RIF might result from controlling lipid metabolism disorders. However, FABP4 $\mathrm{KO}$ is full $\mathrm{KO}$, not specific for kidney tissues. Since the main source of FABP4 is adipose tissue, it is possible that the effects observed in FABP4 KO mice would be consequence of the reduction of FABP4

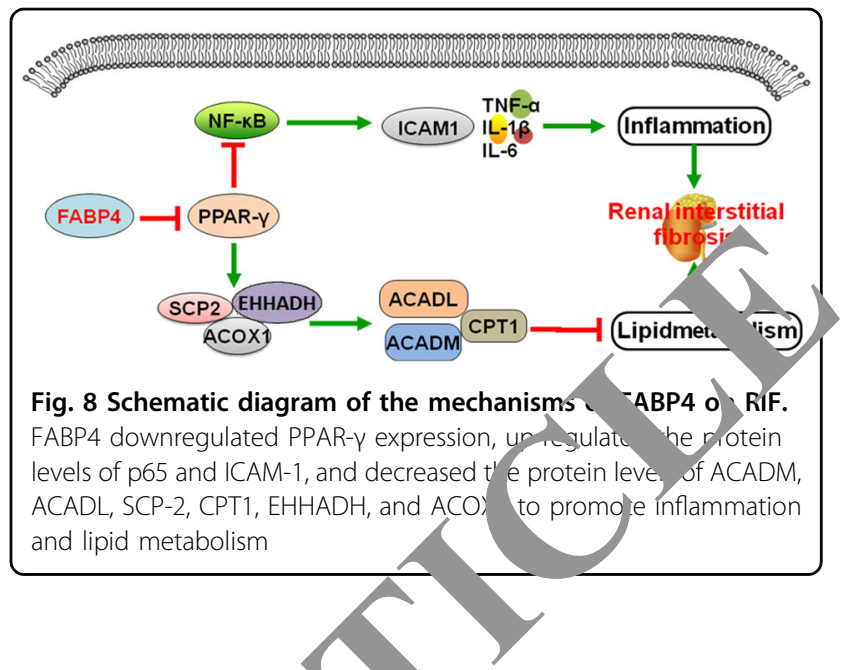

circulating levels found in animals, in addition to the lack of FABP4 spec cally in kidney.

In conclusion deteriorated RIF via promoting inflammation anc inid metabolism disorders (Fig. 8), which sh be considered as one new drug target against RII.

\section{wledgements \\ This was supported by the Key Research and Development Project of iaonin Province (2017225090), the Special Grant for Translational Medicine, ?n Medical University (2015004), the Basic Scientific Research Projects of Lia ring University (No. LF2017010), the Natural Science Foundation of iaoning Province (201602223) and the Project of Leading Talents of Dalian, China.}

\section{Author details}

${ }^{1}$ College of Pharmacy, Dalian Medical University, Western 9 Lvshunnan Road, 116044 Dalian, China. ${ }^{2}$ Department of Pharmacy, The First Affiliated Hospital of Dalian Medical University, 116011 Dalian, China. ${ }^{3}$ Key Laboratory for Basic and Applied Research on Pharmacodynamic Substances of Traditional Chinese Medicine of Liaoning Province, Dalian Medical University, Dalian, China. ${ }^{4}$ National-Local Joint Engineering Research Center for Drug Development (R\&D) of Neurodegenerative Diseases, Dalian Medical University, Dalian, China

Conflict of interest

The authors declare that they have no conflict of interest.

\section{Publisher's note}

Springer Nature remains neutral with regard to jurisdictional claims in published maps and institutional affiliations.

Supplementary information accompanies this paper at (https://doi.org/ 10.1038/s41419-019-1610-5).

Received: 27 November 2018 Revised: 23 April 2019 Accepted: 24 April 2019

Published online: 16 May 2019

\section{References}

1. Gewin, L. S. Renal fibrosis: primacy of the proximal tubule. Matrix Biol. $\mathbf{6 8}$ 248-262 (2018).

2. Deelman, L. \& Sharma, K. Mechanisms of kidney fibrosis and the role of antifibrotic therapies. Curr. Opin. Nephrol. Hypertens. 18, 85-90 (2009). 
3. Howard, C. R. \& Stadler, K. Albumin-bound fatty acids, but not albumin itself, alter mitochondrial bioenergetics in renal proximal tubular cells. Free Radic. Biol. Med. 51, S66-S66 (2011).

4. Allison, S. J. Fibrosis: dysfunctional fatty acid oxidation in renal fibrosis. Nat. Rev Nephrol. 11, 64 (2015).

5. Kang, H. M. et al. Defective fatty acid oxidation in renal tubular epithelial cells plays a key role in kidney fibrosis development. Na.t Med. 21, 37-46 (2015).

6. Eardley, K. S. \& Cockwell, P. Macrophages and progressive tubulointerstitial disease. Kidney Int. 68, 437-455 (2005).

7. Taniguchi, $H$. et al. Involvement of $\mathrm{mcp}-1$ in tubulointerstitial fibrosis through massive proteinuria in anti-gbm nephritis induced in wky rats. J. Clin. Immunol. 27, 409-429 (2007).

8. Hotamisligil, G. S. \& Bernlohr, D. A. Metabolic functions of FABPs-mechanisms and therapeutic implications. Nat. Rev. Endocrinol. 11, 592-605 (2015).

9. $\mathrm{Xu}, \mathrm{H}$. et al. Uncoupling lipid metabolism from inflammation through fatty acid binding protein-dependent expression of UCP2. Mol Cell. Biol. 35, 1055-1065 (2015).

10. Grygiel-Górniak, B. Peroxisome proliferator-activated receptors and their ligands: nutritional and clinical implications-a review. Nutr. J. 13, 17 (2014).

11. Shrestha, U. K. \& Xia, B. Role of peroxisome proliferator activated receptorgamma and its ligands in inflammatory bowel disease. J. Adv. Int. Med. 1, 33-38 (2012).

12. Boss, M., Kemmerer, M., Brüne, B. \& Namgaladze, D. Fabp4 inhibition suppresses ppary activity and vldl-induced foam cell formation in il-4-polarized human macrophages. Atherosclerosis 240, 424-430 (2015).

13. Hsiao, P. J. et al. Pioglitazone enhances cytosolic lipolysis, $\beta$-oxidation and autophagy to ameliorate hepatic steatosis. Sci. Rep. 7, 9030 (2017).

14. Yamamoto, T. et al. Transcriptome and metabolome analyses in exogenous FABP4- and FABP5-treated adipose-derived stem cells. PLoS ONE 11, e0167825 (2016).

15. Duffy, C. M., Xu, H., Nixon, J. P., Bernlohr, D. A. \& Butterick, T. A. Identification of a fatty acid binding protein4-ucp2 axis regulating microglial mediated neuroinfla- mmation. Mol. Cell. Neurosci. 80, 52-57 (2017).

16. Wu, L. E. et al. Identification of fatty acid bind-ing protein 4 ? adipokine that regulates insulin secretion during obesity. Mol. N stab 4 , 465-473 (2014).

17. Hu, B. et al. Fatty acid binding protein-4 (fabp4) is a hypoxia rucicible that sensitizes mice to liver ischemia/reperfusion injury. J. He $/$ 63, 855(2015).

18. Eddy, A. A., Lopez-Guisa, J. M., Okamura, D. M. \& Yam aguchi, I. I. tigating mechan- isms of chronic kidney disease in mouse nodels. Pediatr. Ne hrol. 27, 1233-1247 (2012).

19. Chevalier, R. L., Forbes, M. S. \& Thornhill, B. A. Ur al obstruction as a model of renal interstitial fibrosis and obstructive ne, Kidney Int. 75, 1145-1152 (2009).

20. Xu, L. et al. MiR-125a-5p ameliorates m glycolipid metabolism disorder in type 2 diabetes mellitu hrol.gh targeting of STAT3. Theranostics 8, 5593-560s (2u

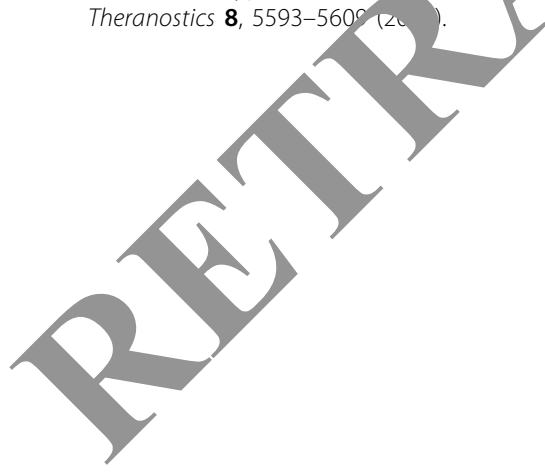

21. Elie, A. et al. Local enrichment of fatty acid-binding protein 4 in the pericardial cavity of cardiovascular disease patients. PLOS ONE 13, e0206802 (2018).

22. Humphreys, B. D. Mechanisms of renal fibrosis. Annu. Rev. Physiol. 7, 10-17 (2012).

23. Chen, J. et al. The metabolic syndrome and chronic kidney disease in U.S. adults. Ann. Int. Med. 140, 167-174 (2004).

24. Lemos, D. R. et al. Interleukin-1 $\beta$ activates a MYC-dependent metabolic switch in kidney stromal cells necessary for progressive tubulointerstitial novisis. J. Am. Soc. Nephrol. 29, 1690-1705 (2018).

25. Yang, C. et al. Chitosan/sirna nanoparticles targeting cyclooxyg type attenuate unilateral ureteral obstruction-induced kidney injury in $\mathrm{T}$. The anostics 5, 110-123 (2015).

26. Lee, E. S. et al. Sarpogrelate hydrochloride ameliorat abetic ne hropathy associated with inhibition of macrophage activit, nd is smatcy reaction in $\mathrm{db} / \mathrm{db}$ mice. PLOS ONE 12, e0179221 (201/).

27. Zhong, C. Q. et al. FABP4 suppresses pro ration and invasion of hepatocellular carcinoma cells and predictc a p prognos; for hepatocellular carcinoma. Cancer Med. 7, 2629-26 018 ).

28. Ge, X. N. et al. FABP4 regulates e inoph cruitment and activation in allergic airway inflammation. Am. J shysiol. Lung "Mol. Physiol. 315, L227-L240 (2018).

29. Bosquet, A. et al. FABP4 mhibito M\$S309403 decreases saturated-fatty-acidinduced endoplasn ticulum ss ss-associated inflammation in skeletal muscle by red ing 8 mapk activation. Biochim. Biophys. Acta 1863, 604-613 (2018).

30. Furuhashi, M. \& Hot ligil, G. S. Fatty acid-binding proteins: role in metabolic diseases nd potential c arug targets. Nat. Rev. Drug Discov. 7, 489-503 (2008).

31. Tan, N. S. an fortive cooperation between fatty acid binding proteins and peroxisome proliry ator-activated receptors in regulating transcription. Mol. Cell. Biol. $2 . \quad 5114-5127$ (2002).

Makowski, L, Brittingham, K. C., Reynolds, J. M., Suttles, J. \& Hotamisligil, G. S. fatty acid-binding protein, aP2, coordinates macrophage cholesterol tring and inflammatory activity. Macrophage expression of aP2 impacts pe oxisome proliferator-activated receptor gamma and ikappab kinase actiJitie. J. Biol. Chem. 280, 12888-12895 (2005).

33. Rosen, E. D. \& Spiegelman, B. M. Ppargamma: a nuclear regulator of metabolism, differentiation, and cell growth. J. Biol. Chem. 276, 37731-37734 (2001).

34. Lefterova, M. I. et al. PPARY and C/EBP factors orchestrate adipocyte biology via adjacent binding on a genome-wide scale. Genes Dev. 22, 2941-2952 (2008).

35. Chen, F. et al. Phosphorylation of ppargamma via active erk1/2 leads to its physical association with p65 and inhibition of NF-kappabeta. J. Cell. Biochem. 90, 732-744 (2003).

36. Abrass, C. K. Lipid metabolism and renal disease. Contrib. Nephrol. 151, 106 (2006).

37. Uysal, K. T., Scheja, L., Wiesbrock, S. M., Bonnerweir, S. \& Hotamisligil, G. S. Improved glucose and lipid metabolism in genetically obese mice lacking aP2. Endocrinology 141, 3388-3396 (2000). 